\title{
Vestibular physical therapy improves turning not straight walking during the inertial sensor- instrumented Timed Up and Go test
}

Kyoung Jae Kim

NASA Johnson Space Center

Yoav Gimmon

University of Haifa

Jennifer Millar

Johns Hopkins University School of Medicine

Kelly Brewer

War Related IIIness and Injury Study Center

Jorge Serrador

Rutgers School of Health Related Professions

Michael Schubert ( $\triangle$ mschube1@jhmi.edu )

Johns Hopkins School of Medicine https://orcid.org/0000-0002-5975-374X

\section{Research}

Keywords: vestibular hypofunction, rehabilitation, gait, turn, timed up and go (TUG), inertial measurement unit (IMU)

Posted Date: July 17 th, 2020

DOl: https://doi.org/10.21203/rs.3.rs-42274/v1

License: (c) (i) This work is licensed under a Creative Commons Attribution 4.0 International License.

Read Full License 
Title: Vestibular physical therapy improves turning not straight walking during the inertial sensor-instrumented Timed Up and Go test

Authors: Kyoung Jae Kim, PhD ${ }^{1,2}$, Yoav Gimmon, PT, $\mathrm{PhD}^{3,4}$, Jennifer Millar, MSPT ${ }^{4,5}$, Kelly Brewer ${ }^{6}$, Jorge Serrador, $\mathrm{PhD}^{7,8}$, Michael Schubert, $\mathrm{PT}, \mathrm{PhD}^{4,5}$

Affiliations:

${ }^{1}$ Human Physiology, Performance, Protection and Operation (H-3PO) laboratory, NASA Johnson Space Center/KBR, Houston, TX, United States

${ }^{2}$ Department of Physical Therapy, University of Miami Miller School of Medicine, Coral Gables, Florida, United States

${ }^{3}$ Department of Physical Therapy, Faculty of Social Welfare \& Health Studies, University of Haifa, Haifa, Israel

${ }^{4}$ Laboratory of Vestibular NeuroAdaptation, Department of Otolaryngology - Head and Neck Surgery, Johns Hopkins School of Medicine, Baltimore, MD, United States

${ }^{5}$ Department of Physical Medicine and Rehabilitation, Johns Hopkins University School of Medicine, Baltimore, MD, United States

${ }^{6}$ Department of Veteran Affairs, Veterans Biomedical Institute, War Related Illness and Injury Study Center, East Orange, NJ, United States

${ }^{7}$ Department of Rehabilitation and Movement Sciences, Rutgers School of Health Professions, Newark, NJ, United States

${ }^{8}$ Department of Pharmacology, Physiology and Neuroscience, Rutgers Biomedical Health Sciences, Newark, NJ, United States 
Corresponding Authors:

Michael C. Schubert, Ph.D.

601 N. Caroline Street, 6th Floor, Baltimore, MD 21287-0910

Phone: 4109557381

Email: mschube1@jhmi.edu

Word count of the text: 3,263

The number of figures and tables in the article: 6 


\section{Abstract}

2 Background: Deficits in vestibular function increase the risk for fall while turning.

3 However, the clinical assessment of turning in patients with vestibular dysfunction is

4 lacking, and evidence is limited that identifies how effective vestibular physical therapy

5 (VPT) is for improving turning performance.

6 Objective: To quantify and compare walking and turning performance during the

7 instrumented timed up and go (TUG) test using inertial measurement units (IMUs) for

8 clinical settings. We investigate novel instrumented TUG parameters for ability to

9 distinguish patients with unilateral vestibular deafferentation (UVD) from control groups,

10 and discriminate the differences in turning parameters of UVD patients following a VPT

11 program.

12 Methods: We recruited 38 patients following UVD surgery, 26 age-matched Veteran

13 patient (VA) controls with reports of non-vestibular dizziness, and 12 age-matched

14 healthy controls. Individuals were donned with body-worn IMUs and given verbal

15 instructions to complete the TUG test as fast as safely possible. The IMU-instrumented

16 and automated assessment of the TUG test provided component-based TUG

17 parameters, including the novel walking:turning ratio. Among the UVD patients, 19

18 patients completed an additional instrumented TUG testing after VPT.

19 Results: The walking:turning time ratio showed that turning performance in pre VPT

20 UVD patients are significantly more impaired than VA patients and healthy controls $(p<$

21 0.001). Vestibular rehabilitation significantly improved turning performance and

22 "normalized" their walking:turning time ratio compared to healthy controls $(p<0.001)$. 
23 However, the duration of the straight walking component in UVD patients before VPT

24 was not significantly different as to that after VPT as well as healthy controls.

25 Conclusions: Our data showed that the IMU-instrumented TUG test can distinguish

26 patients with vestibular deafferentation and objectively quantify the change in their

27 turning performance after surgery. The IMU-based instrumented TUG parameters have

28 potential to quantify the efficacy of VPT and be adopted in the clinic.

29

30 Key Words

31 vestibular hypofunction, rehabilitation, gait, turn, timed up and go (TUG), inertial

32 measurement unit (IMU)

33

34

35

36

37

38

39

40

41

42

43

44

45 


\section{Introduction}

47 Patients with vestibular hypofunction commonly present with dizziness, balance deficits, 48 and gaze disturbance [1]. Such symptoms can cause changes in movement kinematics and compensatory alterations with respect to gait parameters [2]. For example, previous studies have reported significantly reduced head movement during both community

51 ambulation (approximately ten minutes) [3] and during standardized gait testing of short

52 duration [4]. Although such altered movement kinematics may also result in less

53 efficient turning, the current clinical assessment of turning and its related outcome in 54 patients with vestibular dysfunction are not validated. Meta-analysis level data have 55 shown the effectiveness of vestibular physical therapy (VPT) using gaze and gait 56 stability exercises in individuals with vestibular hypofunction [5, 6]. However, the change 57 in turning performance related to the effectiveness of VPT has not been explored. The Timed Up and Go (TUG) test is a reliable measure of functional gait performance

60 that includes walking and turning $180^{\circ}$ as well as transfers to and from a seated position

61 [7]. The ease of administration of the TUG test makes it one of the most commonly 62 utilized measures in VPT [8, 9], and recently, we demonstrated that five weeks of VPT 63 using gaze and gait stability exercises improved the TUG performance despite both pre 64 and post TUG scores being within the clinically accepted normal range [10]. The score 65 from the TUG is simplistic and only considers duration of time and thus lacking an ability 66 to critically evaluate turning performance. To address this limitation, investigators have 67 developed the component-TUG test to individually analyze four unique mobility tasks of 68 the TUG; sit-to-stand and stand-to-sit transitions, straight walking, and turning [11-13]. 
The component-TUG improves the sensitivity to identify pathokinematics, but it's use is dependent on the expertise of a clinician to identify the abnormal kinematics.

72 Recently, inertial tracking technologies have begun to be incorporated into physical

73 therapy clinics, which offers quantitative measurement that has the potential to improve 74 clinical outcomes [14-16]. Body worn wireless inertial measurement units (IMUs) have made objective and quantitative measurements of TUG components possible [17-19]. An instrumented clinical assessment tool must be validated uniquely for its intended patient population, given the unique kinematic differences across pathologies. For example, sit-to-stand performance from a chair during the TUG test is significantly associated with fear of falling in the elderly and an important indicator of overall functioning or balance performance $[20,21]$. In contrast, the measure of sit-to-stand in

81 patients with Parkinson's disease during the component-TUG test was the least reliable component [18]. Recently, it was reported that several outcomes related to both turning

83 and straight-walking components in patients with Parkinson's disease were significantly

84 impaired relative to healthy controls [17-19]. To date, no comparison of turning and

85 straight-walking components has been considered or reported in patients with vestibular 86 hypofunction.

88 Individuals who suffer from vestibular hypofunction often have difficulty turning a corner 89 [22]. During the TUG test, increased time to perform a turn and requiring more steps to 90 complete a turn, are associated with difficulty turning [23]. Since one of the primary

91 functions of the vestibular system is to stabilize the body, examining the motion profiles 
92 of transitions from straight-path walking to turning while walking may provide clues as to

93 why people with vestibular hypofunction have difficulty during turning, particularly

94 relevant given the head velocities can be high [24]. Additionally, there may exist unique

95 relationships between the total time spent straight walking versus turning. In this study,

96 we quantified the walking and turning components via IMUs and introduce novel IMU-

97 based TUG parameters, including the walking:turning time ratio as well as number of

98 steps ratio. The aims of this study were: 1 ) to examine the IMU-instrumented TUG

99 parameters during straight walking and turning in patients following unilateral vestibular

100 deafferentation surgery and two control groups; and 2) to determine the differences

101 between the IMU-instrumented TUG parameters following a progressive five-week VPT

102 program in UVD patients. We hypothesized that reduced time and number of steps to

103 perform a turn might improve after VPT.

104

105 Methods

106 Subjects

107 We enrolled $38(n=22$ female) patient participants after unilateral vestibular

108 deafferentation (UVD) surgery for resection of a vestibular schwannoma. The patients

109 with UVD were $53 \pm 13$ years old. We recruited 26 age-matched Veteran patient

110 controls (VA) ( $n=0$ female) with reports of dizziness not due to vestibular hypofunction

111 based on normal semicircular canal (video head impulse test) and/or otolith function

112 testing (ocular counter roll, vestibular evoked myogenic potential) [25], with a mean age

113 of $56 \pm 12$ years and an additional 12 age-matched healthy control individuals $(n=7$

114 female) with a mean age of $52 \pm 5$ years. There was no significant difference in age 
115 across the three groups $(p=0.606)$. With respect to age, no significant differences in

116 sex were found for UVD patients $(p=0.1323)$ and healthy controls $(p=0.4334)$. Among

117 the UVD patients, 19 patients $(\mathrm{n}=11$ female) completed an additional instrumented

118 TUG test after completing VPT, mean duration of $68 \pm 45$ days later. The study was

119 approved by the Johns Hopkins University and the East Orange VA Healthcare System

120 IRBs. Informed consent was obtained from each individual.

122 Test procedure and data acquisition

123 Participants completed questionnaires regarding demographic information. The body

124 worn sensor system that we currently use is comprised of five wireless MTw IMUs,

125 Xsens shirt, and Velcro straps (Xsens Technologies, Netherlands) [26-28]. In this study,

126 acceleration and angular velocities along three perpendicular axes data were analyzed

127 from three IMUs (chest and each ankle) during the TUG test. The IMU sampling

128 frequency was $100 \mathrm{~Hz}$ and data were transmitted to the Awinda Station (Xsens

129 Technologies Netherland) before being saved to a tablet PC via USB

130 interface. Specifically, the chest IMU was placed inside the pocket of an Xsens shirt on

131 the right border of the sternum. The ankle IMUs were attached to the ankle just above

132 the lateral malleolus using Velcro straps. Participants were asked to perform three trials

133 of the TUG. The participants were given verbal instructions to stand up from an initially

134 seated position, walk three meters as fast as safely possible, cross a line marked on the

135 floor, turn around a cone, walk back, and sit down. Once testing was completed,

136 participants were assisted with doffing the sensor system. 
Data processing and instrumented TUG variables

139 The TUG test consists of five consecutive tasks; standing up from a chair, straight

140 walking, $180^{\circ}$ turning, straight walking, and turning to sitting down on the chair [7]. We

141 analyzed the signal from the ankle IMUs and the chest IMU to segment in two

142 components, i.e., straight walking and turning. The chest IMU gyroscope data for yaw

143 rotation provided a turning direction (e.g. clockwise and counter-clockwise) and an

144 easily distinguished trace for the initiation of turning from a straight-path. Since the time

145 derivative of the turn-angle is the angular velocity, we calculated the trunk angular

146 displacement in the horizontal (yaw) plane by integrating the yaw angular velocity. The

147 mathematical model-based turning segmentation [18] provided the ability to

148 automatically distinguish performance of the individual tasks of the TUG (e.g., straight

149 walking and $180^{\circ}$ turning around a cone). We applied the gait event detection algorithm

150 to the pitch angular velocity waveform recorded from both ankle IMUs. The mid-swing

151 event was selected to monitor step counts [29]. Figure 1 shows the segmented phases

152 of straight walking (yellow) and turning (green) and the detected gait event (red circle:

153 mid-swing). The end of trunk rotation was marked with a couple of steps after the

154 second straight walking component (the second yellow shaded area) and denoted the

155 end of the second turn component that occurs as participants return to seated position

156 on the chair. Both the first standing up from a chair and the second turning (returning to

157 sit on the chair) components were excluded from this below analysis. 
161 This technique was used to determine the TUG variables, which included total time

162 (sec), total number of steps, straight walking time (sec), number of steps straight

163 walking, turning time ( $\mathrm{sec}$ ), and number of steps turn-walking. It is often observed in the

164 clinical setting that a person can ambulate fairly well during the straight-path

165 component, only to slow or modify their gait way while performing the turn. Although the

166 clinician instructs the individual to walk as fast as safely possible during the TUG test,

167 individuals walk with different speeds ranging from slow to fast. Therefore, we also

168 determined the ratio of time period and the number of steps a participant required for

169 individuals to walk straight compared to the time period and the number of steps

170 needed to perform the $180^{\circ}$ turn. The walking:turning ratios provides a method of

171 normalizing across the unique and varied patterns of quality of turning motion.

173 Statistical Analysis

174 Statistical analysis was performed using SPSS (version 26, Chicago, IL, USA) software.

175 All variables were normally distributed hence, parametric analysis was performed. A one-way ANOVA was performed to compare variables between groups (UVD patients

177 vs VA patient controls vs healthy controls). Post hoc testing using the least significant

178 difference (LSD) correction was applied to compare between groups differences. To

179 evaluate the effect of VPT on the TUG parameters a paired t-test was performed to

180 compare the UVD patients' pre-test results vs. the post-test results. A second analysis

181 between the UVD patients' post-test results and the healthy controls was conducted by

182 independent t-test. The level of statistical significance was set at $p \leq 0.05$. Mean and

183 one standard deviation (1 SD) values of the dependent variables were calculated for 
184 each of the test components (straight walking time and steps numbers; turning time and

185 steps numbers; and total time and steps numbers). Descriptive statistics (mean and 1

186 SD) were used to summarize the results.

187

188

189

190

191

192

193

194

195

196

197

198

200

201

202

203

204

205 healthy and VA controls was $50 \%$ of the straight walking time.

\section{Results}

Between Groups lesioned ear in the patients with UVD.

Table 1 presents the comparison result of the TUG variables between groups. We found no difference in any of the kinematic variables for turns towards or away from the

Time period variables: The VA controls with dizziness walked significantly slower during the straight component of the TUG compared to UVD patients ( $25 \%$ slower, $p<0.001)$ and healthy controls $(32 \%$ slower, $p<0.001)$. During the turning component of the TUG, healthy controls were significantly faster than both the VA controls ( $35 \%$ faster, $p<$ $0.001)$ and UVD patients ( $30 \%$ faster, $p<0.001)$. The total time to complete the TUG test were significantly different from each other, with the control group completing it in the shortest duration, followed by the UVD patients ( $15 \%$ slower than healthy controls) then the VA controls (33\% slower than healthy controls). In the UVD patients before VPT, the turning time was $61 \%$ of the straight walking while the turning time in both 
207 Number of steps variables: The VA controls used $15 \%$ more steps during the straight

208 component of the TUG compared to the healthy controls $(p=0.004)$, while the UVD

209 patients trended to also use more steps $(9 \%, p=0.053)$ than the healthy controls.

210 During the turning component of the TUG, however, the UVD patients used a $16 \%$

211 greater number of steps, than both the VA patient controls $(p<0.001)$ and the healthy

212 controls $(p=0.005)$. The healthy control group used fewer steps during the entire TUG

213 test compared with either UVD patients $(12 \%$ less steps, $p=0.011)$ but not the VA

214 controls $(10 \%$ less steps, $p=0.055)$.

215

216 Walking:turning ratio variables: The VA controls had a significantly higher ratio of

217 walking:turning steps $(30 \%$ higher, $p<0.001)$ compared with both the UVD patients and

218 healthy controls $(22 \%$ higher, $p<0.001)$. Only the UVD patients had a significantly

219 reduced ratio of time spent in walking:turning $(p<0.001,19 \%$ reduction than both VA

220 and healthy controls).

222 Effect of Vestibular Rehabilitation

223 The UVD patients significantly improved after five weeks of VPT in most of the

224 measured TUG parameters. Figure 2 shows an example of the Pre and Post-rehab

225 results in a patient with UVD. The number of steps during the straight walking

226 component before VPT was the same as that after VPT (8 steps). During $180^{\circ}$ turning

227 around a cone, the UVD patient 'total number of steps' reduced from five to three,

228 Figure 2. The duration of the straight walking component before and after VPT were

$2293.92(s)$ and $3.65(s)$, respectively $(p=0.06)$. However, after VPT, the turning time was 
230 decreased from $2.35(\mathrm{~s})$ to $1.81(\mathrm{~s}),(\mathrm{p}<0.001)$. The walking:turning step ratio after VPT

231 was increased to 2.7 from 1.6 before VPT $(p<0.001)$. After VPT, the walking:turning

232 time ratio was also increased to 2.1 from 1.7 before VPT $(p<0.001)$.

233

234

[Figure 2 here]

235

236 Table 2 shows walking and turning related variables to identity the effect of VPT.

[Table 2 here]

240 Time period variables: During the straight walking component of the TUG, the UVD

241 patients walked $9 \%$ faster (albeit insignificant improvement, $p=0.06$ ) during post-test

242 evaluation of the straight component of the TUG compared to baseline. However, during

243 the turning component of the TUG, the UVD patients were significantly faster post-rehab

$244(27 \%$ faster, $p<0.001)$. Additionally, the total time to complete the TUG test was

245 significantly shorter after VPT than pre-rehab $(13 \%$ faster, $p<0.001)$.

247 Number of steps variables: During the straight walking component of the TUG, the UVD

248 patients used less steps after VPT, though as above, this was not significant ( $5 \%$ less

249 steps, $p=0.145)$. However, during the turning component of the TUG, the UVD patients

250 used significantly less steps after VPT ( $36 \%$ less steps, $p<0.001)$. The UVD patients

251 also used significantly fewer steps completing the TUG after VPT compared to pre-

$252 \operatorname{rehab}(15 \%$ less steps, $p<0.001)$. 
254 Walking:turning ratio variables: The UVD patients increased significantly the ratio of

255 walking:turning steps compared to pre-rehab $(28 \%$ increased, $p<0.001)$. Similarly, the

256 UVD patients significantly increased the ratio of time spent in walking:turning (18\%

257 increased, $p<0.001)$ post-rehab.

258

259 Comparing the UVD patients' post-test results to healthy controls, the data suggests the

260 UVD patients 'normalized' their timing strategies of movement during the TUG test

261 given none of the timing variables were different than the healthy controls $(0.1<p<$

2620.361 ) (Figure 3). However, the spatial properties of the UVD patients were less altered

263 given, the UVD patients used $10 \%$ more steps during the straight component of the

264 TUG compared to healthy controls $(p=0.007)$. During the turning component of the

265 TUG however, the UVD patients used $12 \%$ less steps than healthy controls $(p=0.011)$

266 after VPT. Thus, the UVD patients walking:turning steps ratio was $28 \%$ significantly

267 higher than the healthy controls (Figure 3). Finally, after VPT, the UVD patients' ratio of

268 turning time was $50 \%$ of their straight walking time, similar with both healthy and VA

269 controls (see the Between Groups section).

270

[Figure 3 here]

272

273

Discussion

274 A clinical assessment tool must be valid, accurate, and reliable within the intended

275 patient group if it is going to be clinically useful. Poor sensitivity of a clinical tool leads to 
276 problems with data analysis and interpretation and an unreliable assessment of the

277 efficacy of rehabilitation programs. The TUG is a well-known clinical test of mobility and

278 fall risk with the virtue of being a quick and simple assessment [7-9]. However, recent

279 studies have revealed the limitations of the TUG test and there is conflicting information

280 and opinion about interpreting TUG test results [30-33]. The clinical TUG test measures

281 time, not considering any change in kinematics during various transitions between

282 components. To overcome this limitation, the component-TUG and its

283 instrumented version using IMUs have been suggested and identified as having the

284 ability to classify fallers among the elderly [11, 12], amputee [13], and Parkinson's

285 disease populations [17-19]. However, to the best of our knowledge, only one study has

286 incorporated IMUs to examine the test-retest reliability of the instrumented TUG in

287 patients with vestibular disorders and its association with fall risk [34]. The authors do

288 suggest that the instrumented TUG has potential to enable clinicians and therapists to

289 objectively assess the efficacy of their interventions in patients with vestibular disorders.

290 However, they did not use the instrumented TUG to examine the effectiveness of VPT

291 in patients with vestibular hypofunction; furthermore, no parameters have been derived

292 from IMUs to comparatively analyze the turning sub-component of the TUG in

293 association with straight walking.

295 Ours is the first study to distinguish patients with vestibular pathology from healthy 296 controls and non-vestibular dizzy patient controls based on the ratio between walking 297 and turning while completing the instrumented TUG. This is a critical result as it has 298 been reported that typical measures of gait, such as the 10-meter walking test, may not 
299 provide consistent results in screening patients with vestibular impairments [10, 35-38].

300 Our data also support this observation given the UVD patients showed no differences in

301 straight walking time before or after completing VPT. However, the UVD patients did 302 demonstrate abnormally increased walking:turning ratios due to significantly slower

303 turning, compared against two control groups. It is also interesting to note that, after

304 VPT, the UVD patients also demonstrated reduced turning time which was half of the

305 straight walking time-similar to the VA and healthy controls. Only the UVD patient

306 group before showed that the turning time was $61 \%$ of the straight walking. Our finding

307 suggests the percentage of time spent turning (50\%) compared with straight-walking

308 may be a clinically meaningful parameter for distinguishing patients with vestibular

309 hypofunction as well as assessing the effectiveness of VPT in those patients.

311 Vestibular disorders cause changes in gait behavior [39]; however, the explicit kinematic

312 differences in turning by those with vestibular deficit remains unclear. Furthermore,

313 while there are extensive studies showing how effective VPT is for reducing dizziness

314 and falls in patients with vestibular dysfunction $[5,6,10]$, the contributions of VPT as a

315 treatment for reducing turning difficulties are less understood. In healthy controls,

316 turning $180^{\circ}$ around a cone during the TUG involves a smooth and continuous top-down

317 rotation from the head to the trunk $[40,41]$ with resulting asymmetries in gait

318 parameters between limbs (i.e. stride length and stance time) [42-43]. It is presumed

319 that head movement and upper body coordination during transitions from straight-path

320 walking to turns is critical to ensure a stable position and to aid in gaze stabilization [44-

$32146]$. In contrast, deficits in vestibular function cause various disturbances in spatial 
322 orientation, gait, head movement, and upper body coordination. It was recently reported

323 that patients with unilateral vestibular hypofunction reveal fewer, smaller, and slower

324 head movements after surgery [3, 4]. Additionally, these authors suggested that early

325 referral for vestibular rehabilitation may be beneficial to improve the recovery of gait,

326 dynamic stability, head movement, and upper body coordination. Our results related to

327 turning performance improvement demonstrated that completing five weeks of the VPT

328 (gaze and gait stability exercises) might restore the normal coordination between the

329 head and upper body.

$331 \quad$ Limitations

332 Our data revealed the short-term effects of completing VPT on turning in patients with

333 vestibular deafferentation surgery, it is unknown whether this improvement persists.

334 Patients with other causes for vestibular hypofunction may display different results.

335 Additionally, we did not compare outcomes against multi-segmental coordination from

336 the upper body which may reveal additional compensatory strategies. Future research

337 is needed to fully understand the potential benefits of vestibular rehabilitation on head

338 movement and upper body coordination during turning.

340 Conclusions

341 The findings of this study suggest the commonly used clinical version of the TUG test

342 can be instrumented to distinguish patients with surgical vestibular deafferentation and

343 identify improvement of turning ability after vestibular rehabilitation. 


\section{List of Abbreviations}

346 IMU: inertial measurement unit, LSD: least significant difference, SD: standard

347 deviation, TUG: timed up and go, UVD: unilateral vestibular deafferentation, VA:

348 Veteran Affair, VPT: vestibular physical therapy.

350 Ethics approval and consent to participate

351 The study was approved by the Johns Hopkins University Ethics Committee (Reference 352 Number: IRB00059430) and the VA New Jersey Health Care Systems and the Human

353 Research Protection Office of the Department of Defense (Reference Number: 01386),

354 titled "Sensorimotor Assessment and Treatment of Vestibular Dysfunction." An informed 355 consent was obtained from all the participants of the study.

356

357 Consent for publication

358 Not applicable.

360 Availability of data and materials

361 The datasets generated during and/or analyzed during the current study are not publicly 362 available due to privacy laws and other restrictions but are available from the 363 corresponding author on reasonable request.

\section{Competing interests}


366 All authors declare that there is no proprietary, financial, professional or other personal

367 interest of any nature or kind in any product, service or company that could be

368 construed as influencing the position presented in this study.

370 Funding

371 The author(s) disclosed receipt of the following financial support for the research,

372 authorship, and/or publication of this article: This work was supported in part by the

373 Department of Defense under the Neurosensory and Rehabilitation Research Award

374 Program (Grant Award Number: W81XWH-15-1-0442).

375

\section{Authors' contributions}

377 Kyoung Jae Kim: Conceptualization, Methodology, Software, Data curation, Writing -

378 original draft, Writing - review \& editing, Visualization. Yoav Gimmon: Validation, Formal

379 analysis, Data collection, Writing - original draft, Writing - review \& editing. Jennifer

380 Millar: Data collection, Writing - review \& editing. Kelly Brewer: Data collection, Writing -

381 review \& editing. Jorge Serrador: Data collection, Writing - review \& editing. Michael

382 Schubert: Investigation, Resources, Writing - review \& editing, Supervision, Project

383 administration, Funding acquisition. All authors read and approved the manuscript.

385 Acknowledgements

386 The authors wish to thank the volunteers for participating in this study. 


\section{References}

1. Burzynski J, Sulway S, and Rutka J. Vestibular rehabilitation: review of indications, treatments, advances, and limitations. Curr Otorhinolaryngol Rep. 2017;5(3):160-166.

2. Herdman SJ. Advances in the treatment of vestibular disorders. Phys Ther. 1997; 77(6):602-618.

3. Paul SS, Dibble LE, Walther RG, Shelton C, Gurgel RK, Lester ME. Characterization of head-trunk coordination deficits after unilateral vestibular hypofunction using wearable sensors. JAMA Otolaryngol Head Neck Surg. 2017;143(10):1008-1014 .

4. Paul SS, Dibble LE, Walther RG, Shelton C, Gurgel RK, Lester ME. Reduced purposeful head movements during community ambulation following unilateral vestibular Loss. Neurorehabil Neural Repair. 2018;32(4-5):309-316.

5. Hillier S, Mcdonnell M. Is vestibular rehabilitation effective in improving dizziness and function after unilateral peripheral vestibular hypofunction? Eur J Phys Rehabil Med. 2016;52(4):541-556.

6. McDonnell MN, Hillier SL. Vestibular rehabilitation for unilateral peripheral vestibular dysfunction. Cochrane Database Syst Rev. 2015;1(1):1465-1858.

7. Podsiadlo D, Richardson S. The timed "Up \& Go": a test of basic functional mobility for frail elderly persons. J Am Geriatr Soc. 1991;39(2):142-148.

8. Donoghue OA, Savva GM, Cronin H, Kenny RA, Horgan NF. Using timed up and go and usual gait speed to predict incident disability in daily activities among 
community-dwelling adults aged 65 and older. Arch Phys Med Rehab. 2014;95(10):1954-1961.

9. Whitney SL, Marchetti GF, Schade A, Wrisley DM. The sensitivity and specificity of the Timed" Up \& Go" and the Dynamic Gait Index for self-reported falls in persons with vestibular disorders. J Vestib Res. 2004;14(5):397-409.

10. Millar JL, Gimmon Y, Roberts D, Schubert MC. Improvement after vestibular rehabilitation not explained by improved passive VOR gain. Front Neurol. 2020;11(79):1-9.

11. Wall JC, Bell C, Campbell S, Davis J. The timed get-up-and-go test revisited: measurement of the component tasks. J Rehabil Res Dev, 2000;37(1):109-114.

12. Vernon S, Paterson K, Bower K, McGinley J, Miller K, Pua YH, Clark RA. Quantifying individual components of the timed up and go using the kinect in people living with stroke. Neurorehabil Neural Repair. 2015;29(1):48-53.

13. Clemens SM, Gailey RS, Bennett CL, Pasquina PF, Kirk-Sanchez NJ, Gaunaurd IA. The component timed-up-and-go test: the utility and psychometric properties of using a mobile application to determine prosthetic mobility in people with lower limb amputations. Clin Rehabil. 2018;32(3):388-397.

14. Cuesta-Vargas Al, Galán-Mercant A, Williams JM. The use of inertial sensors system for human motion analysis. Phys Ther Rev. 2010;15(6):462-473.

15. Horak F, King L, Mancini M. Role of body-worn movement monitor technology for balance and gait rehabilitation. Phys Ther. 2015;95(3):461-470. 
16. Wang Q, Markopoulos P, Yu B, Chen W, Timmermans A. Interactive wearable systems for upper body rehabilitation: a systematic review. J Neuroeng Rehabil. 2017;14(1):1-21.

17.Zampieri C, Salarian A, Carlson-Kuhta P, Aminian K, Nutt JG, Horak FB. The instrumented timed up and go test: potential outcome measure for disease modifying therapies in Parkinson's disease. J Neurol Neurosurg Psychiatry. 2010;81(2):171-176.

18. Salarian A, Horak FB, Zampieri C, Carlson-Kuhta P, Nutt JG, Aminian K. iTUG, a sensitive and reliable measure of mobility. IEEE Trans Neural Syst Rehabil Eng. 2010;18(3):303-310.

19. Palmerini L, Mellone S, Avanzolini G, Valzania F, Chiari L. Quantification of motor impairment in Parkinson's disease using an instrumented timed up and go test. IEEE Trans Neural Syst Rehabil Eng. 2013;21(4):664-673.

20. Deshpande N, Metter EJ, Bandinelli S, Lauretani F, Windham BG, Ferrucci L. Psychological, physical, and sensory correlates of fear of falling and consequent activity restriction in the elderly. Am J Phys Med Rehabil. 2008;87(5):354-362.

21.Zijlstra W1, Bisseling RW, Schlumbohm S, Baldus H. A body-fixed-sensor-based analysis of power during sit-to-stand movements. Gait Posture. 2010;31(2):272278.

22. Agrawal Y, Ward BK, Minor LB. Vestibular dysfunction: prevalence, impact and need for targeted treatment. J Vestibul Res-Equil. 2013;23(3):113-117. 
23. Orendurff MS, Segal AD, Berge JS, Flick KC, Spanier D, Klute GK. The kinematics and kinetics of turning: limb asymmetries associated with walking a circular path. Gait Posture. 2006;23(1):106-111.

24. Forsell C, Conradsson D, Paquette C, Franzén E. Reducing gait speed affects axial coordination of walking turns. Gait Posture. 2017;54(1):71-75.

25. Serrador JM, Lipsitz LA, Gopalakrishnan GS, Black FO, Wood SJ. Loss of otolith function with age is associated with increased postural sway measures. Neurosci Lett. 2009;465(1):10-15.

26. Kim KJ, Gimmon Y, Sorathia S, Beaton KH, Schubert MC. Exposure to an extreme environment comes at a sensorimotor cost. npj Microgravity. 2018;4(1):1-8.

27. Gimmon Y, Migliaccio AA, Kim KJ, Schubert MC. VOR adaptation training and retention in a patient with profound bilateral vestibular hypofunction. Laryngoscope. 2019;129(11):2568-2573.

28. Kim KJ, Gimmon Y, Millar J, Schubert MC. Using Inertial Sensors to Quantify Postural Sway and Gait Performance during the Tandem Walking Test. Sensors. 2019;19(4):751 .

29. Allseits EK, Agrawal V, Prasad A, Bennett C, Kim KJ. Characterizing the impact of sampling rate and filter design on the morphology of lower limb angular velocities. IEEE Sensors J. 2019;19(11):4115-4122.

30. Barry E, Galvin R, Keogh C, Horgan F, Fahey T. Is the Timed Up and Go test a useful predictor of risk of falls in community dwelling older adults: a systematic review and meta-analysis. BMC Geriatr. 2014;14(1):1-14. 
31. Beauchet O, Fantino B, Allali G, Muir SW, Montero-Odasso M, Annweiler C. Timed Up and Go test and risk of falls in older adults: a systematic review. J Nutr Health Aging. 2011;15(10):933-938.

32. Boulgarides LK, McGinty SM, Willett JA, Barnes CW. Use of clinical and impairment-based tests to predict falls by community-dwelling older adults. Phys Ther. 2003;83(4):328-339.

33. Schoene D, Wu SM, Mikolaizak AS, Menant JC, Smith ST, Delbaere K, Lord SR. Discriminative ability and predictive validity of the timed up and go test in identifying older people who fall: systematic review and meta-analysis. J Am Geriatr Soc. 2013;61(2):202-208.

34. Sankarpandi SK, Baldwin AJ, Ray J, Mazzà C. Reliability of inertial sensors in the assessment of patients with vestibular disorders: a feasibility study. BMC Ear Nose Throat Disord. 2017;17(1):1-9.

35. Allum JH, Scheltinga A, Honegger $F$. The effect of peripheral vestibular recovery on improvements in vestibulo-ocular reflexes and balance control after acute unilateral peripheral vestibular loss. Otol Neurotol. 2017;38(10):531-538.

36. Cohen HS, Mulavara AP, Peters BT, Sangi-Haghpeykar H, Bloomberg JJ. Tests of walking balance for screening vestibular disorders. J Vestib Res. 2012;22(2):95-104.

37. Tramontano M, Bergamini E, losa M, Belluscio V, Vannozzi G, Morone G. Vestibular rehabilitation training in patients with subacute stroke: A preliminary randomized controlled trial. NeuroRehabilitation. 2018;43(2):247-254. 
38. Rastoldo G, Marouane E, Mahmoudi NE, Péricat D, Bourdet A, Timon-David E, Olivier D, CHABBERT C, Tighilet B. Quantitative evaluation of a new posturolocomotor phenotype in a rodent model of acute unilateral vestibulopathy. Front Neurol. 2020;11(505):1-19.

39. Bent LR, McFadyen BJ, Inglis JT. Vestibular contributions during human locomotor tasks. Exerc Sport Sci Rev. 2005;33(3):107-113.

40. Hollands MA, Ziavra NV, Bronstein AM. A new paradigm to investigate the roles of head and eye movements in the coordination of whole-body movements. Exp Brain Res. 2004;154(2):261-266.

41. Taylor MJD, Dabnichki P, Strike SC. A three-dimensional biomechanical comparison between turning strategies during the stance phase of walking. Hum Mov Sci. 2005;24(4):558-573.

42. Hase K, Stein RB. Turning strategies during human walking. J Neurophysiol. 1999;81(6):2914-2922.

43. Thigpen MT, Light KE, Creel GL, Flynn SM. Turning difficulty characteristics of adults aged 65 years or older. Phys Ther. 2000;80(1):1174-1187.

44. Crane BT, Demer JL. Human gaze stabilization during natural activities: translation, rotation, magnification, and target distance effects. J Neurophysiol. 1997;78(4):2129-2144.

45. Demer JL, Viirre ES. Visual-vestibular interaction during standing, walking, and running. J Vestib Res. 1996; 6(4):295-313.

46. Mulavara AP, Bloomberg JJ. Identifying head-trunk and lower limb contributions to gaze stabilization during locomotion. J Vestib Res. 2002;12(5):255-269. 
Figure 1. An example of the segmented TUG phases (straight walking and turning) and the detected gait event (mid-swing).

Figure 2. Comparison between pre and post TUG performances. Top: pre VPT, Bottom: post VPT. See Figure 1 legend. Note that both Pre and Post VPT TUG total time scores would be considered as normal.

Figure 3. Comparison of walking:turning ratio parameters (error bar: 95\% confidence interval). * Significant difference between groups. 
Table 1. Between groups results: mean \pm SD (95\% confidence interval).

\begin{tabular}{|c|c|c|c|}
\hline Parameter & $\begin{array}{c}\text { UVD } \\
(\mathrm{N}=38)\end{array}$ & $\begin{array}{l}\text { VA Controls } \\
(\mathrm{N}=26)\end{array}$ & $\begin{array}{c}\text { Healthy Controls } \\
(\mathrm{N}=12)\end{array}$ \\
\hline Straight Walking Time (s) & $\begin{array}{c}3.6 \pm 0.87 \\
(3.48-3.81)\end{array}$ & $\begin{array}{c}4.5 \pm 1.16^{*} \\
(4.24-4.76)\end{array}$ & $\begin{array}{c}3.4 \pm 0.56 \ddagger \\
(3.19-3.61)\end{array}$ \\
\hline Turn Walking Time (s) & $\begin{array}{l}2.2 \pm 0.53 t \\
(2.15-2.36)\end{array}$ & $\begin{array}{c}2.3 \pm 0.49 \\
(2.23-2.45)\end{array}$ & $\begin{array}{c}1.7 \pm 0.31 \ddagger \\
(1.67-1.91)\end{array}$ \\
\hline Total TUG Time (s) & $\begin{array}{c}5.9 \pm 1.27 \dagger \\
(5.66-6.14)\end{array}$ & $\begin{array}{c}6.8 \pm 1.49^{\star} \\
(6.51-7.18)\end{array}$ & $\begin{array}{c}5.1 \pm 0.82 \ddagger \\
(4.88-5.49)\end{array}$ \\
\hline Straight Walking \# of Steps & $\begin{array}{c}7.4 \pm 1.90 \dagger \\
(7.10-7.82)\end{array}$ & $\begin{array}{c}7.8 \pm 1.41 \\
(7.51-8.15)\end{array}$ & $\begin{array}{c}6.8 \pm 1.09 \ddagger \\
(6.39-7.21)\end{array}$ \\
\hline Turn Walking \# of Steps & $\begin{array}{c}4.3 \pm 1.21 \dagger \\
(4.15-4.61)\end{array}$ & $\begin{array}{c}3.7 \pm 0.87^{*} \\
(3.53-3.93)\end{array}$ & $\begin{array}{c}3.7 \pm 0.62 \\
(3.53-4.00)\end{array}$ \\
\hline Total TUG \# of Steps & $\begin{array}{c}\mathbf{1 1 . 8} \mathbf{\pm 2 . 8 3 \dagger} \\
(11.31-12.37)\end{array}$ & $\begin{array}{c}11.5 \pm 1.97 \\
(11.12-12.01)\end{array}$ & $\begin{array}{c}10.5 \pm 1.50 \ddagger \\
(10.01-11.13)\end{array}$ \\
\hline Walking:Turning Time Ratio & $\begin{array}{c}\mathbf{1 . 6} \pm \mathbf{0 . 3 5 \dagger} \\
(1.57-1.71)\end{array}$ & $\begin{array}{c}1.9 \pm 0.43^{*} \\
(1.84-2.04)\end{array}$ & $\begin{array}{c}1.9 \pm 0.26 \\
(1.82-2.02)\end{array}$ \\
\hline Walking:Turning Steps Ratio & $\begin{array}{c}1.7 \pm 0.41 \\
(1.67-1.82)\end{array}$ & $\begin{array}{c}2.2 \pm 0.52^{*} \\
(2.06-2.30)\end{array}$ & $\begin{array}{c}1.8 \pm 0.31 \ddagger \\
(1.71-1.94)\end{array}$ \\
\hline
\end{tabular}

One-way ANOVA comparison is significant for all variables.

* Significant difference between UVD patients and VA controls.

† Significant difference between UVD patients and healthy controls. ¥ Significant difference between VA controls and healthy controls.

Based on a post hoc tests with LSD correction for between groups comparisons. 
Table 2. Effect of VPT pre-post results in 19 UVD patients: mean \pm SD (95\% confidence interval).

\begin{tabular}{|c|c|c|c|}
\hline Parameter & UVD Pre-Rehab & UVD Post-Rehab & $P$ value \\
\hline Straight Walking Time (s) & $\begin{array}{c}3.9 \pm 0.95 \\
(3.65-4.13)\end{array}$ & $\begin{array}{c}3.6 \pm 0.63 \\
(3.50-3.82)\end{array}$ & $P=0.06$ \\
\hline Turn Walking Time (s) & $\begin{array}{c}2.3 \pm 0.51 \\
(2.16-2.43)\end{array}$ & $\begin{array}{c}1.8 \pm 0.39 \\
(1.76-1.96)\end{array}$ & $P<0.001$ \\
\hline Total TUG Time (s) & $\begin{array}{c}6.2 \pm 1.34 \\
(5.85-6.54)\end{array}$ & $\begin{array}{c}5.5 \pm 0.93 \\
(5.28-5.74)\end{array}$ & $P<0.001$ \\
\hline Straight Walking \# of Steps & $\begin{array}{c}7.9 \pm 2.18 \\
(7.40-8.54)\end{array}$ & $\begin{array}{c}7.5 \pm 1.16 \\
(7.28-7.86)\end{array}$ & $P=0.145$ \\
\hline Turn Walking \# of Steps & $\begin{array}{c}4.5 \pm 1.46 \\
(4.19-4.96)\end{array}$ & $\begin{array}{c}3.3 \pm 0.62 \\
(3.23-3.54)\end{array}$ & $P<0.001$ \\
\hline Total TUG \# of Steps & $\begin{array}{c}12.5 \pm 3.45 \\
(11.63-13.47)\end{array}$ & $\begin{array}{c}10.9 \pm 1.38 \\
(10.61-11.32)\end{array}$ & $P<0.001$ \\
\hline Walking:Turning Time Ratio & $\begin{array}{c}1.7 \pm 0.33 \\
(1.63-1.81)\end{array}$ & $\begin{array}{c}2.01 \pm 0.34 \\
(1.92-2.09)\end{array}$ & $P<0.001$ \\
\hline Walking:Turning Steps Ratio & $\begin{array}{c}1.8 \pm 0.33 \\
(1.70-1.88)\end{array}$ & $\begin{array}{c}2.3 \pm 0.52 \\
(2.17-2.44)\end{array}$ & $P<0.001$ \\
\hline
\end{tabular}


Figures

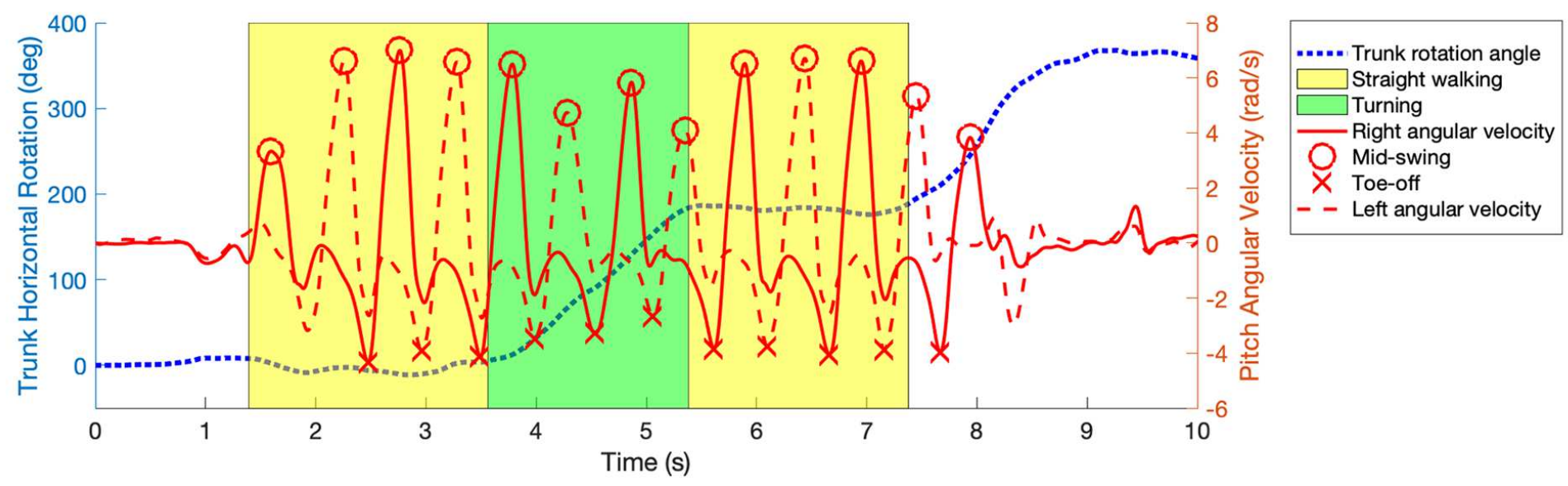

Figure 1

An example of the segmented TUG phases (straight walking and turning) and the detected gait event (mid-swing).

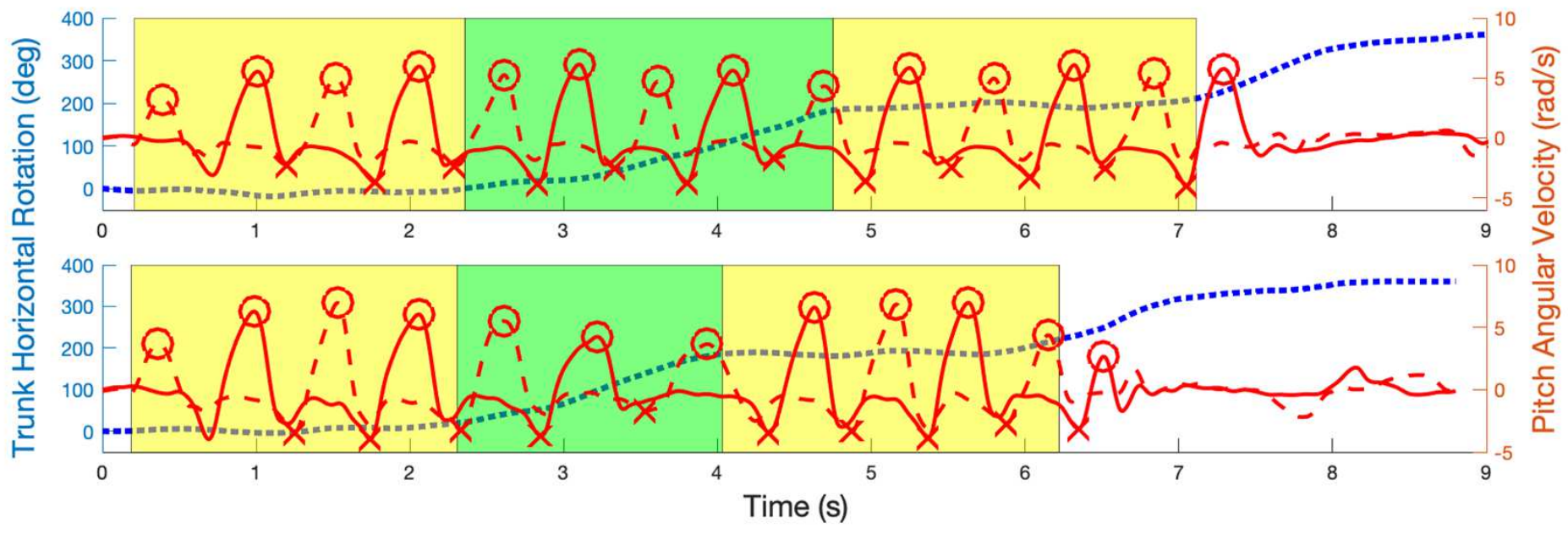

Figure 2

Comparison between pre and post TUG performances. Top: pre VPT, Bottom: post VPT. See Figure 1 legend. Note that both Pre and Post VPT TUG total time scores would be considered as normal. 


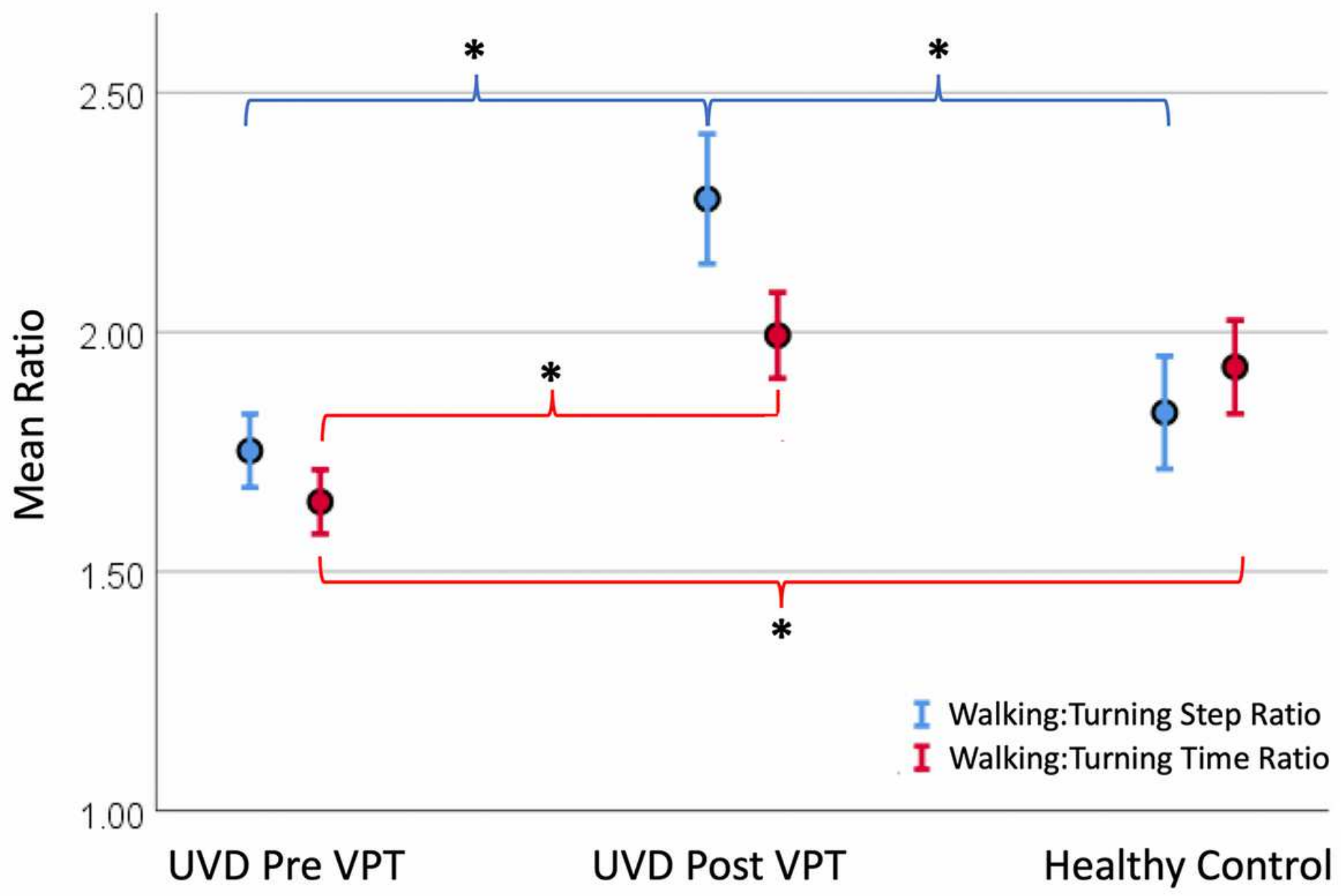

Figure 3

Comparison of walking:turning ratio parameters (error bar: 95\% confidence interval). * Significant difference between groups. 